\title{
Ocular shedding of herpes simplex virus
}

\author{
S B Kaye, N Madan, T C Dowd, C A Hart, K McCarthy, A Patterson
}

\begin{abstract}
The presence, frequency, and relationship of ocular and oral shedding of herpes simplex virus to previous herpes keratitis was investigated. This was to determine if a history of herpes keratitis predisposes to shedding of the herpes simplex virus into the tear film and/or mouth. Swabs were collected from the eyes and mouth of two groups of patients thrice weekly over a two- to four-month period. Group A comprised nine patients with a history of herpes labialis, group B 15 patients with a history of herpes keratitis. Herpes simplex virus type 1 was isolated from $1 \cdot 33 \%$ of mouth specimens but was not identified in any of the ocular specimens. There was no significant difference between groups $A$ and $B$ in terms of ocular or oral shedding. Oral shedding appears to be independent of a previous herpes keratitis. The tear film is an unlikely source of virus in persons either with no history of herpes keratitis or between attacks in those patients with a history of previous herpes keratitis.
\end{abstract}

Since Buddingh et al ${ }^{1}$ demonstrated herpes simplex virus (HSV) in the saliva and stools of asymptomatic persons, there have been several well documented reports on the shedding of HSV into saliva. ${ }^{2-6}$ While both spontaneous and induced ocular HSV shedding in animals is well established, ${ }^{7}$ the situation in humans remains largely unexplored. In 1967 Kaufman et al. ${ }^{2}$ studied a group of patients with and without a history of HSV labialis (HSVL) and found an incidence of asymptomatic ocular shedding of between $0.79 \%$ and $1.4 \%$. In addition they found $\mathrm{HSV}$ in the tears and saliva of experimentally infected animals in the absence of a herpetic lesion. ${ }^{2}$ There have been no subsequent reports on humans corroborating Kaufman's findings, nor attempts to establish if an association exists between previous herpetic eye disease and subsequent ocular and or oropharyngeal shedding.

The aim of this study was to establish the presence and frequency of ocular shedding, its relationship to previous HSV ophthalmia (HSVO), and the relationship between ocular and oropharyngeal shedding.

St Paul's
Liverpool
S B Kaye

TC Dowd

A Patterson

\section{Department of}

Microbiology, University

of Liverpool

N Madan

C A Hart

K McCarthy

Correspondence to: $\mathrm{Mr}$ S B Kaye, St Paul's Eye Hospital, Old Hall Street, Liverpool L3 9PF.

Accepted for publication 23 August 1989 only clinical manifestation of HSV was recurrent herpes labialis, with an average frequency of 2.9 episodes per year (range 1-6 episodes per year).

Group B. This comprised 15 healthy patients
(10 males, five females). The average age was 49 years (range 19-69). Fourteen patients had experienced previous dendritic keratitis which had been treated at this hospital by a combination of debridement and topical acycloguanosine or idoxuridine (used in only one case). The remaining patient had had a herpetic blepharitis. All patients except three had suffered from recurrent HSVL to give an average frequency of 1.9 episodes per year (range $0-6$ episodes per year). Seven patients had had only one clinical episode of dendritic keratitis. The remaining seven patients had had recurrent dendritic keratitis, with two patients having had a subsequent disciform keratitis necessitating the use of topical steroids. The average frequency of recurrent ocular herpes was 0.135 episode per year (range $0.05-0.35$ episode per year). The time between the resolved ocular herpetic lesion and entry into the study was 1.5 weeks for 10 patients, and between 1 year and 20 years for the remaining five patients.

\section{STUDY DESIGN AND SPECIMEN}

COLLECTION

The study was conducted from October through January 1988-9. Patients had specimens collected on Monday, Wednesday, and Friday of each week. Informed consent was obtained from all patients.

Tear film swabs. Preocular tear film was collected by rotation of a swab along the lower fornix to the medial canthus, where it was held to absorb tears for 10 seconds. Care was taken to avoid the corneal epithelium. The 15 patients comprising group B had swabs taken from their previously affected eye. The nine patients comprising group A had swabs taken from each eye. They were, however, placed in only one bottle of transport medium and hence defined as one swab.

Oropharyngeal swabs. A swab was rotated along the buccal mucosa and over the mucosa of the hard palate.

All swabs were immediately placed in transport medium and kept on ice prior to inoculation. For group A the transport medium was a 1 $\mathrm{ml}$ solution phosphate buffered saline (PBS A) supplemented with $100 \mathrm{U} / \mathrm{ml}$ of penicillin, 100 $\mu \mathrm{g} / \mathrm{ml}$ of streptomycin (P/S), and $2 \%$ fetal calf serum (FCS). For group B a sterile transport medium pack was used (Virocult, UK).

In addition two of the nine patients in group $A$, patients $A 3$ and $A 5$, rinsed their mouth with a $5 \mathrm{ml}$ collutary of maintenance medium containing medium 199 with Earle's salts (E-199) (Gibco, UK), supplemented with 2\% FCS and $2 \%$ sodium bicarbonate. The 15 patients in group B had their eyes examined on each visit by slit-lamp biomicroscopy, and all patients had their oropharnyx examined with a torch and 
tongue depressor for mouth ulceration. In addition the patients were asked to record any other events such as HSVL, upper respiratory tract infections (URTI), and dental procedures.

\section{VIRUS ISOLATION}

Virus was detected within each specimen by the inoculation of specimen fluid into tissue culture tubes containing confluent monolayered Vero cells (Flow Laboratories) previously propagated in growth medium (E-199 supplemented with $5 \%$ FCS, $2 \%$ sodium bicarbonate, and $\mathrm{P} / \mathrm{S}$ ). Each tube contained $15 \times 10^{4}$ cells $/ \mathrm{ml}$ growth medium prepared $48 \mathrm{~h}$ prior to inoculation to allow formation of a monolayer. At inoculation the growth medium overlying the cells was discarded, and $0.2 \mathrm{ml}$ of each specimen was inoculated in duplicate on to the monolayered Vero cells. As a control two test tubes were inoculated with transport medium alone.

Tubes were then kept on rockers at $37^{\circ} \mathrm{C}$ for one hour, thus allowing viral adsorption, and were re-fed with $0.8 \mathrm{ml}$ of maintenance medium. Cultures were incubated on stationary racks at $37^{\circ} \mathrm{C}$ and re-fed every other day with maintenance medium and $50 \mathrm{U} / \mathrm{ml}$ nystatin. Viral cytopathic effects (CPE) were recorded daily for two weeks under an inverted light microscope. On detection of viral CPE, incubation was allowed to continue until $100 \% \mathrm{CPE}$ was obtained. Virus was then harvested from these cultures and stored at $-70^{\circ} \mathrm{C}$.

Isolates were then typed with an immunofluorescent monoclonal antibody (Syva MicroTrak, USA).

\section{STATISTICS}

Statistical analysis was by means of a FisherYates exact probability test and a Poisson distribution. $\mathrm{P}<0.05$ was considered significant.

\section{Results}

In total 752 mouth and 752 eye swabs were collected (Table I). There were no eye isolations. Ten mouth swabs were positive for HSV type 1 $(1.33 \%$ of swabs). None of the patients developed detectable or symptomatic intraoral or eye lesions during the study. There were 19 episodes of HSVL during the study of which two $(10.5 \%)$ were concurrent with a mouth isolation. Conversely, of the 10 mouth isolations two (20\%) were concurrent with HSVL.

GROUP A

Of the mouth and eye swabs 379 were from this

TABLE I Isolation of HSV from the eye and mouth

\begin{tabular}{llcll}
\hline & No of swabs & $\begin{array}{l}\text { Mouth } \\
\text { isolations }\end{array}$ & $\begin{array}{l}\text { Eye } \\
\text { isolations }\end{array}$ & $\begin{array}{l}\text { No of } H S V L \\
\text { during study }\end{array}$ \\
\hline Group A & 379 & 7 & 0 & $11(2)$ \\
Group B & 373 & 3 & 0 & $8(0)$ \\
Total & 752 & 10 & 0 & $19(2)$ \\
\hline
\end{tabular}

*The figures in brackets refer to the incidence of HSVL lesions which were concurrent with a mouth isolation. group, to give an average of 42 swabs per patient (range 16-51).

Mouth specimens. Seven swabs were positive for HSV-1 ( $1 \cdot 85 \%$ of swabs): five isolations were from patient A5 (10\% of his swabs), and two from patient A4 (4.5\% of his swabs). None of these isolations were in clusters. Five of the mouth rinses from patient A5 were positive for HSV type 1 (these were concurrent with the positive mouth swabs from this patient).

None of the mouth rinses from patient A3 were positive. Two of the isolations were associated with a concurrent episode of HSVL (28.6\% of isolations). Three isolations were associated with a URTI, leaving two isolations not associated with any event.

Eye specimens. There were no isolations.

Associated events. There were 11 episodes of HSVL (range 0-3), of which two were associated with a mouth isolation $(18 \cdot 2 \%)$. There were two episodes of an URTI without concurrent HSVL, which were associated with three of the isolations.

\section{GROUP B}

A total of 373 swabs were collected from this group to give an average of 25 swabs per patient (range 13-42).

Mouth specimens. There were three isolations ( $0.8 \%$ of the 373 swabs): one was from patient B7 ( $5 \%$ of his swabs) and two from B2 (6.25\% of his swabs). None of the isolations were associated with any event.

Eye specimens. There were no isolations.

Associated events. There were eight episodes of HSVL and one of URTI, none of which were associated with an isolation.

\section{GROUP A AND B COMPARED}

Although the average number of swabs per patient was higher in group $A(42 \cdot 1)$ than in group B (24.9), the compliance rate (actual number of attendances/expected number of attendances) was on average above $80 \%$ for both groups. Whereas a patient in group A attended on average for 14 weeks, a patient in group B attended on average for eight weeks before leaving the study. There was no significant difference in the number of mouth isolations between groups A and B (Fisher-Yates exact test, $\mathrm{p}=0 \cdot 35$ )

There was no significant difference in the number of eye isolations from either group; it was zero for both groups. A Poisson distribution was used to assess further the significance of the results. The results from those patients with mouth isolations were then tested using a mean of $10 / 752 \mathrm{~S}$ ( $\mathrm{S}$ is the number of swabs taken from the patient under question) for a significantly high number of mouth isolations. Only patient A5 had a significantly high number of mouth isolations (Poisson distribution, $\mathrm{p}=0.0006$ ).

Group A was then compared with group B without the inclusion of patient A5. There was still no significant difference in the number of mouth isolations between the two groups (Fisher-Yates exact test, $\mathrm{p}=0.56$ ). 


\section{Discussion}

This study confirms the findings of previous authors in that aymptomatic shedding of HSV can occur in the mouth. ${ }^{1-6}$ Although the frequency of oral shedding in this study is less than that of Spruance $(7 \cdot 6 \%),{ }^{3}$ Douglas and Couch (4.6\%), ${ }^{5}$ Buddingh et al (7\%), ${ }^{1}$ and Hatherley et al $(9.6 \%),{ }^{6}$ it is similar to that of Kaufman et al $(1 \cdot 5 \%) .{ }^{2}$ When Buddingh and colleagues' series is considered for adults only, the frequency is decreased to $2 \cdot 5 \% .^{\prime}$ The lower frequency of mouth shedding is unlikely to have been due to the use of swabs, as the isolation of HSV in the two patients who had concurrent swabs and mouth rinses was identical. In additon care was taken to swab the mucosa of the hard palate. ${ }^{89}$

All the isolations in this study occurred as solitary events - that is, the minimum interval between isolations was two weeks. In Spruance's study only $38 \%$ of isolations occurred as solitary events, with the remaining $62 \%$ occurring in clusters. ${ }^{3}$ Thus frequency of isolations in his study which occurred as solitary events was $2 \cdot 8 \%$, which is commensurate with our findings.

None of the patients that we studied had dental procedures. This factor, in addition to a lower frequency of recurrent HSVL $(2 \cdot 3 / \mathrm{yr}$ as compared with $4 \cdot 4 / \mathrm{yr}$ reported in Spruance's study $^{3}$ ) may have contributed to the lower frequency of mouth isolations. There was no significant difference in the prevalence of mouth shedding between groups $\mathrm{A}$ and $\mathrm{B}$; thus the rate of asymptomatic oral shedding is independent of previous herpetic keratitis. Reactivation and subsequent shedding in the mouth can occur with a herpes labialis lesion but is largely independent of it. The numbers of mouth isolations that were concurrent with a HSVL lesion $(20 \%)$ was similar to those previously reported. ${ }^{34}$

HSV was not isolated from the tear film of patients with or without a history of herpetic keratitis, and so with respect to ocular shedding groups A and B are identical. That is, a previous history of herpes keratitis does not appear to predispose to the shedding of HSV into the tear film in the asymptomatic state.

It is unlikely that the absence of detectable shedding was due to the use of swabs as opposed to an eye bath, as there is no difference in the detection rate between these two techniques. The effect of treatment, however, during the previous acute attacks needs to be considered; 14 patients in group B had their dendritic ulcers treated by a combination of mechanical debridement and topical acycloguanosine. It has been shown that this combination tends to reduce the recurrence rate following primary ocular herpes, while a lower rate of isolation of HSV from corneal discs has been demonstrated in patients treated with acycloguanosine. ${ }^{1011}$ These factors may have had a role in the absence of ocular shedding in this study.

The significance of oral shedding in relation to HSVO is unknown. A person who is a mouth shedder would probably have been shedding and expectorating virus for some time. But the risk of a mouth shedder or their consort developing HSV keratitis is at present unknown, and to answer this would require a long term prospective study.

The absence of ocular shedding as demonstrated in this study would suggest that the tear film is an unlikely source of virus in persons either with no history of herpetic keratitis or between attacks in those patients with a previous history of herpes keratitis.

1 Buddingh CJ, Schrum DI, Lanier JC, Guidry DJ. Studies of the natural history of herpes simplex infections. Pediatrics 1953; 11: 595-609.

2 Kaufman HE, Brown DC, Ellison EM. Recurrent herpes in the rabbit and man. Science 1967; 156: 1628-9.

3 Spruance SL. Pathogenesis of herpes simplex labialis: excretion of virus in the oral cavity. $\mathcal{F}$ Clin Microbiol 1984; 19 675-9.

4 Spruance SL, Overall JC Jr, Kern ER, Krueger GC, Pliam V, Miller W. The natural history of recurrent herpes simplex labialis. Implications for antiviral therapy. $N E n g l, \mathcal{H}$ Med 1977; 297: 69-75.

5 Douglas RG Jr, Couch RB. A prospective study of chronic herpes simplex virus infection and recurrent herpes labialis in humans. F Immunol 1970; 104: 289-95.

6 Hatherley LI, Hayes K, Jack I. Herpes virus in an obstetric hospital. II. Asymptomatic virus excretion in staff members. hospital. II. Asymptomatic

7 Hill JM, Dudley JB, Shimomura Y, Kaufman HE. Quantitation and kinetics of induced HSV-l ocular shedding. Cur Eye Res 1986; 5: 241-6.

8 Scott TFM, Steigman AJ, Convey JH. Acute infectious gingivostomatitis. FAMA 1941; 117: 999-1005.

9 Weathers DR, Griffin JW. Intraoral ulcerations of recurrent herpes simplex and recurrent apthae: two district clinical entities. 7 Am Dent Assoc 1970; 81: 81-8.

10 Easty DL, Shimeld C, Claoue CMP, Menage M. Herpes simplex virus isolation in chronic stromal keratitis: human and laboratory studies. Curr Eye Res 1987; 6: 69-74.

11 Shimona Y, Ohashi Y, Maeda N. Herpes keratitis therapy to reduce recurrence. Curr Eye Res 1987; 6: 105-10. 